ARTICLE

DOI: $10.1038 / \mathrm{s} 41467-017-00576-7$

\title{
An engineered bispecific DNA-encoded IgG antibody protects against Pseudomonas aeruginosa in a pneumonia challenge model
}

Ami Patel ${ }^{1}$, Antonio DiGiandomenico ${ }^{2}$, Ashley E. Keller², Trevor R. F. Smith³, Daniel H. Park' ${ }^{3}$ Stephanie Ramos $^{3}$, Katherine Schultheis (10 ${ }^{3}$, Sarah T. C. Elliott ${ }^{1}$, Janess Mendoza ${ }^{3}$, Kate E. Broderick ${ }^{3}$, Megan C. Wise $^{1}$, Jian Yan $^{3}$, Jingjing Jiang ${ }^{3}$, Seleeke Flingai ${ }^{1}$, Amir S. Khan ${ }^{3}$, Kar Muthumani ${ }^{1}$, Laurent Humeau ${ }^{3}$, Lily I. Cheng ${ }^{2}$, Leslie Wachter-Rosati ${ }^{2}$, C. Kendall Stover (1) ${ }^{2}$, Niranjan Y. Sardesai ${ }^{3}$ \& David B. Weiner ${ }^{1}$

The impact of broad-spectrum antibiotics on antimicrobial resistance and disruption of the beneficial microbiome compels the urgent investigation of bacteria-specific approaches such as antibody-based strategies. Among these, DNA-delivered monoclonal antibodies (DMAbs), produced by muscle cells in vivo, potentially allow the prevention or treatment of bacterial infections circumventing some of the hurdles of protein IgG delivery. Here, we optimize DNAdelivered monoclonal antibodies consisting of two potent human IgG clones, including a nonnatural bispecific lgG1 candidate, targeting Pseudomonas aeruginosa. The DNA-delivered monoclonal antibodies exhibit indistinguishable potency compared to bioprocessed IgG and protect against lethal pneumonia in mice. The DNA-delivered monoclonal antibodies decrease bacterial colonization of organs and exhibit enhanced adjunctive activity in combination with antibiotics. These studies support DNA-delivered monoclonal antibodies delivery as a potential strategy to augment the host immune response to prevent serious bacterial infections, and represent a significant advancement toward broader practical delivery of monoclonal antibody immunotherapeutics for additional infectious pathogens.

\footnotetext{
${ }^{1}$ The Wistar Institute of Anatomy \& Biology, Philadelphia, PA 19104, USA. ${ }^{2}$ Medlmmune, Gaithersburg, MD 20878, USA. ${ }^{3}$ Inovio Pharmaceuticals, Plymouth Meeting, PA 19462, USA. Ami Patel and Antonio DiGiandomenico contributed equally to this work. Correspondence and requests for materials should be addressed to D.B.W. (email: dweiner@wistar.org)
} 
T he impact of growing antimicrobial resistance (AMR) arising from broad-spectrum antibiotic exposure threatens the antibiotic cornerstone of modern medicine. The global rate of mortality from AMR infections is estimated to be $>700$ 000/year and expected to rise over the next decade ${ }^{1}$, and the CDC reports over 2 million AMR cases in the United States each year (>23 000 deaths/year) $)^{2}$. The lack of progress in the development of new antibiotic classes and new understanding of host-pathogen interactions is prompting interest in microbiome-friendly approaches that engage the immune system with minimal disruption of the normal microbiota. One such approach is the investigation of antibody technology for preventing and treating bacterial infections. Therapeutic monoclonal antibodies (mAbs) are now a mainstay to treat several diseases, including primary immunodeficiencies, autoimmune disorders, asthma, cancer, and graft transplantation (reviewed in refs. ${ }^{3-8}$ ). However, only two mAbs are approved for treatment of infectious diseases in humans, palivizumab (targeting respiratory syncytial virus) and bezlotoxumab (targeting Clostridium difficile toxin B). Raxibacumab and obiltoxaximab, both targeting inhalation anthrax, were approved under the Food and Drug Administration (FDA) animal rule because they could not be reasonably tested for efficacy in humans. Several other infectious disease $\mathrm{mAb}$ candidates are currently being evaluated in human clinical trials (clinicaltrials.gov, reviewed in refs 9,10 ). Yet, there are several technological hurdles with cost-effective mAb-based delivery that impede a paradigm shift from broad-spectrum approaches to pathogen-specific, targeted prevention and treatment of bacterial infections. These limitations to protein IgG administration include dosage (in the range of $\mathrm{mg} / \mathrm{kg}$ ), $\mathrm{mAb}$ production challenges, and significant costs associated with current manufacturing technology.

DNA plasmid delivery offers a novel approach to circumvent the hurdles of traditional $\mathrm{mAb}$ delivery through direct, in vivo immunoglobulin (Ig) production. DNA-delivered monoclonal antibodies (DMAb) are transiently expressed and secreted by skeletal muscle cells and directly enter the systemic circulation to prevent virulence while promoting bacterial clearance. This is facilitated though specific Ig heavy and light chain nucleotide sequence optimizations, formulation development, and by recent advances in in vivo electroporation (EP) technology that has directly lead to increased DNA uptake into cells, translating to significantly enhanced DNA expression in vivo ${ }^{11}$.

Employing this pathogen-specific approach we utilized the DMAb platform to deliver two well-characterized mAbs targeting Pseudomonas aeruginosa, an environmentally ubiquitous bacterium that can cause life-threatening infections in critically ill and immunocompromised patients. In addition, intrinsic antibiotic resistance via elaborate drug efflux systems, the acquisition of antibiotic resistance through horizontal gene transfer, as well as the ability to form biofilms makes $P$. aeruginosa a formidable foe in seriously ill patients. Two promising $\mathrm{mAb}$ targets against $P$. aeruginosa include the Psl exopolysaccharide and the type 3 secretion (T3S) injectisome PcrV protein. Psl is a highly abundant serotype-independent polysaccharide important for host cell attachment, biofilm formation, and immune evasion $^{12-14}$. The T3S injectisome induces intoxication by injection of multiple effectors directly into the host cell cytoplasm where they interact with a multitude of eukaryotic co-factors. $\mathrm{PcrV}$ is thought to sit at the apex of the molecular syringe and plays a prominent role, along with other bacterial proteins, in host cell membrane pore formation ${ }^{15}$. Protective mAbs to both targets were recently described, with each alone exhibiting potent activity in multiple $P$. aeruginosa infection models ${ }^{16}$, 17 . In addition, clinical candidate MEDI3902, a bispecific mAb targeting Psl and PcrV, was recently described and shown to exhibit synergistic protective activity when compared to the individual mAbs or a parental mAb mixture ${ }^{18}$. DMAb delivery as an alternative strategy to bypass the need for protein IgG could be highly beneficial for administration of pathogen-specific mAbs to at-risk target populations. Furthermore, the low cost of DMAb production, the favorable safety profile of DNA delivery in humans, and the ease of administration ${ }^{19}$ would benefit a wider population of individuals that are at risk for infection.

Here, we describe the development and analysis of synthetic DMAbs consisting of a monospecific anti-PcrV IgG (DMAb$\alpha \mathrm{PcrV})$ and clinical candidate bispecific antibody MEDI3902 (DMAb-BiSPA) for in vivo production and activity. We observe that DMAb production in vivo can rapidly yield functional and protective titers for both constructs. These DMAbs can persist and have similar potency to bioprocess-produced mAbs, along with comparable prevention of $P$. aeruginosa colonisation of major organs. We also demonstrate that skeletal muscle cells can support production and secretion of functional, engineered bispecific IgG. This finding highlights the flexibility of the host cellular machinery to form novel, non-natural Ig forms in vivo and expands the possibilities for encoding additional pathogenicspecific affinities. Furthermore, we show that DMAb-BiSPA exhibits enhanced protective activity with antibiotic treatment in a lethal pneumonia model. Taken together, our results suggest that DNA delivery of full length IgG mAbs is a promising strategy for prevention of serious bacterial infections and possibly for other therapeutic indications.

\section{Results}

Design and in vitro expression of anti-P. aeruginosa DMAbs. We selected two anti- $P$. aeruginosa $\mathrm{mAb}$ genes to be re-encoded for optimal expression into a DNA expression vector system based on their previously described potent protective in vivo activity against lethal $P$. aeruginosa infection ${ }^{17}, 18$. The nucleotide and amino acid sequences of the human immunoglobulin gamma 1 (IgG1) heavy and light chains (Fab and Fc portions) were optimized taking into consideration both human and mouse codon bias, and encoded as a single, polycistronic unit in the pGX0001 DNA plasmid backbone, resulting in two constructs: DMAb- $\alpha$ PcrV and DMAb-BiSPA (Fig. 1a). The heavy and light chains are expressed as a single mRNA transcript and then cleaved post-translationally at a porcine teschovirus-1 2A (P2A) cleavage site. A furin cleavage site (RGRKRRS) was also included to ensure complete removal of the P2A from the final in vivo produced antibody.

We first assessed the ability for each construct to express full length human IgG1 antibody following in vitro transfection of HEK293T cells. The DMAb-transfected cells and supernatants were harvested after $48 \mathrm{~h}$ and a total IgG enzyme-linked immunosorbent assay (ELISA) was performed on the cell lysates and in the medium, which verified DMAb IgG production and secretion (Fig. 1b). A Western blot was also performed to confirm that both antibody heavy and light chains were expressed. The heavy chain for the bispecific DMAb runs at a higher molecular weight as it encodes two variable region specificities (Fig. 1c). pGX0001 DNA vector was included as a negative control and purified anti-PcrV IgG1 as a positive control.

Expression of DMAb- $\alpha$ PcrV and DMAb-BiSPA in mice. Following confirmation of in vitro expression, we investigated expression of DNA-delivered DMAb- $\alpha$ PcrV and DMAb-BiSPA in mice. To confirm DMAb expression in mouse muscle, anti- $P$. aeruginosa DMAb- $\alpha \mathrm{PcrV} \quad(100 \mu \mathrm{g})$, DMAb-BiSPA (100 $\mu \mathrm{g})$, control DMAb-DVSF3 $(100 \mu \mathrm{g})$, or control pGX0001 empty vector $(100 \mu \mathrm{g})$ were administered to $\mathrm{BALB} / \mathrm{c}$ mice by 


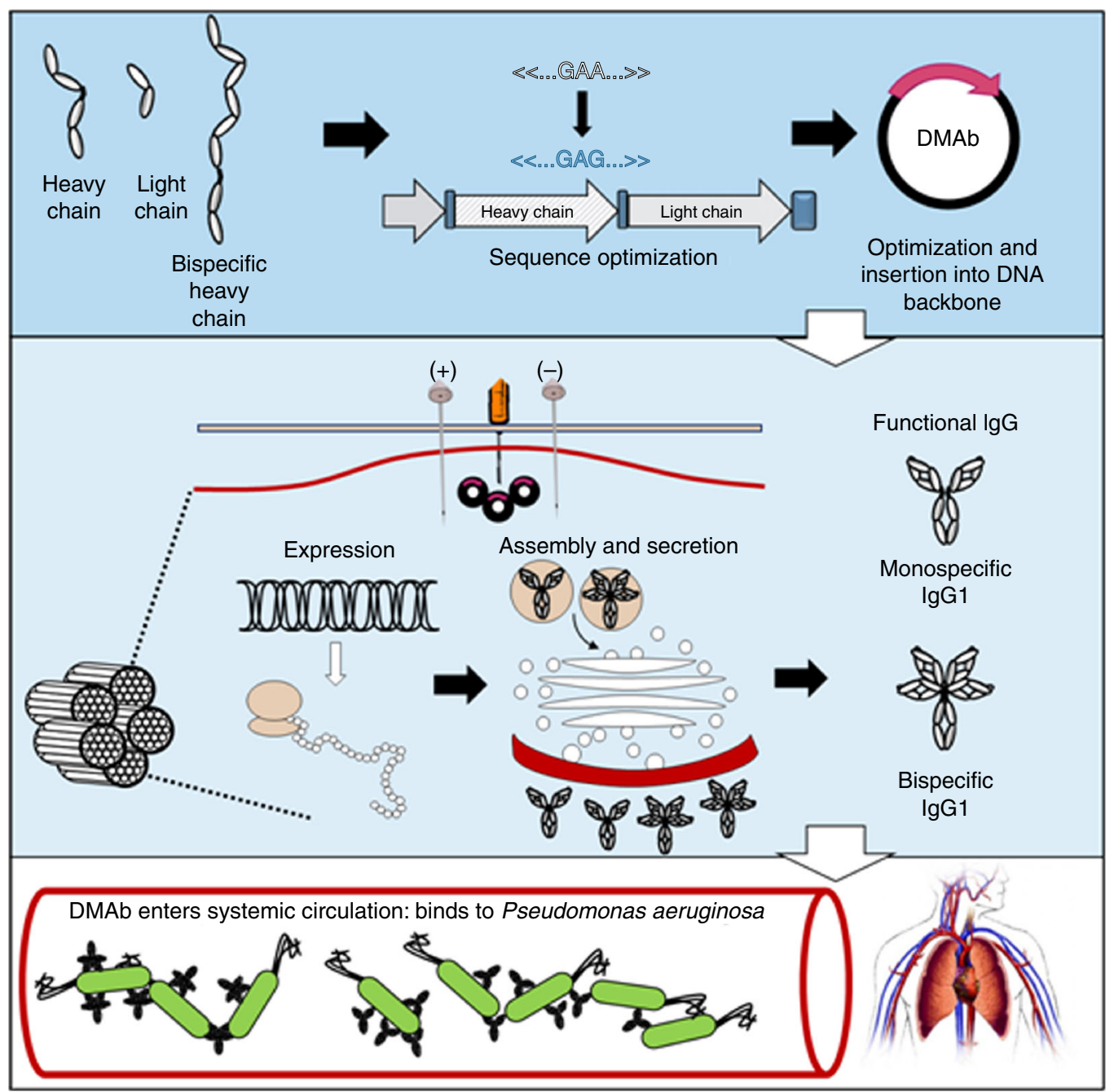

b DMAb expression in
293T cell supernatant

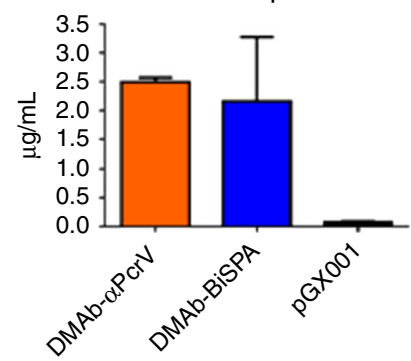

DMAb expression in 293T cell lysate

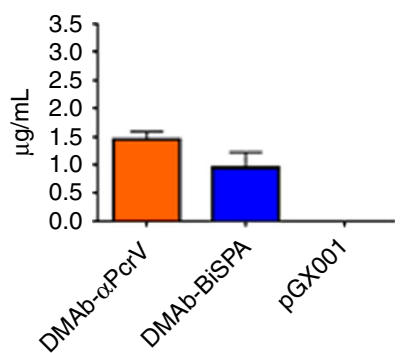

C

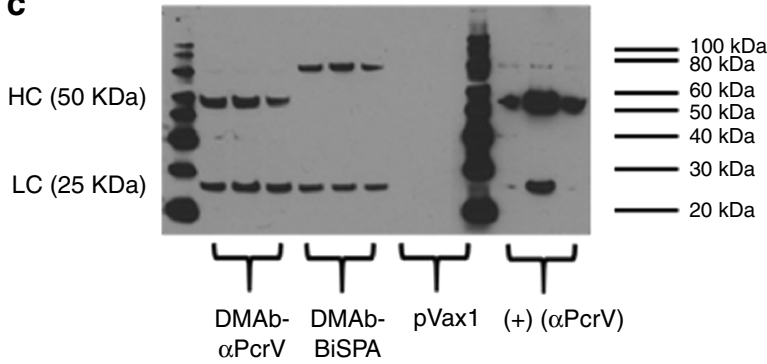

Fig. 1 Schematic diagram of DMAb delivery and in vitro expression. a DMAbs were designed to encode IgG antibody heavy and light chains of monoclonal antibody clones V2L2MD and MEDI3902, resulting in the DMAb- $\alpha$ PcrV and DMAb-BiSPA constructs. The optimized DMAb constructs are administered to mice by in vivo IM-EP, and muscle cells being to synthesize an produce mAb. Fully functional DMAb is secreted and enters the systemic circulation. (The image of the human circulatory system is licensed under a Creative Commons Share-Alike 3.0 License (CC-BY-SA) https://creativecommons.org/ licenses/by-sa/3.0/ and can be found at thecardiovascularsystem.wikispaces.com/HOME). b HEK $293 \mathrm{~T}$ cells were transfected with $1 \mu \mathrm{g} /$ well of DMAb- $\alpha$ PcrV, DMAb-BiSPA, or control pGX0001. Supernatant and cell lysates were harvested after $48 \mathrm{~h}$. Samples were assayed for human lgG. Error bars represent the standard deviation. c A Western blot was performed with cell lysates from transfected cells. $10 \mu \mathrm{g}$ total cell lysate was loaded in each lane and run on an SDS-PAGE gel, followed by transfer onto a nitrocellulose membrane. The membrane was probed with a goat anti-human lgG $\mathrm{H}+\mathrm{L}$ antibody, conjugated to HRP. Samples were developed using an ECL chemiluminescence kit and visualized on film

intramuscular (IM) injection in the tibialis anterior (TA), followed by intramuscular electroporation (IM-EP). Muscle tissue was harvested 3 days post injection and sections were probed with a goat anti-human IgG Fc antibody followed by detection with a donkey anti-goat IgG conjugated to AF488 (Fig. 2). Following confirmation of expression in vivo, we performed further experiments to assay DMAb levels in systemic circulation. Human IgG1 induces an anti-antibody response in immunocompetent mice, since it is recognized as non-self by the murine immune system. Therefore, we first evaluated expression in 

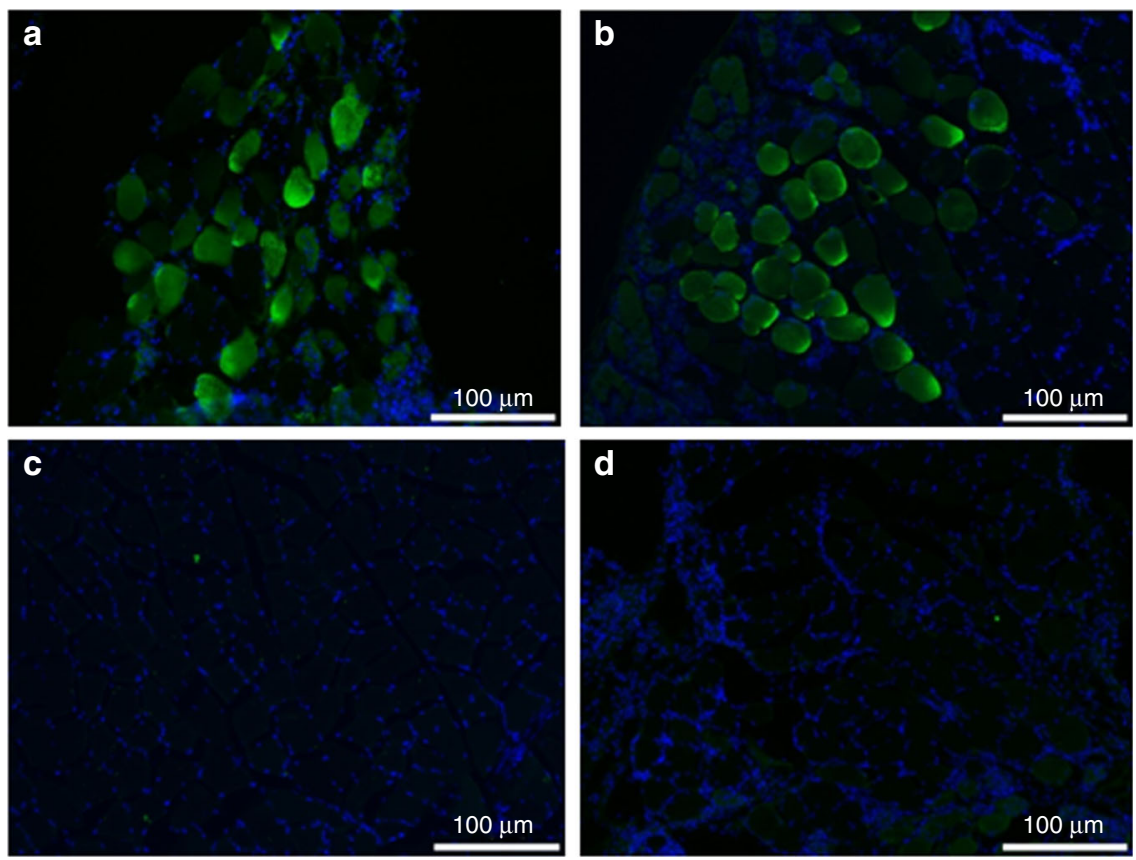

Fig. 2 Expression of DMAb- $\alpha$ PcrV and DMAb-BiSPA in mouse skeletal muscle. BALB/c mice received a DNA injection, in the TA muscle with DMAb- $\alpha$ PcrV or DMAb-BiSpA DNA followed by in vivo EP. a DMAb- $\alpha$ PcrV, b DMAb-BiSPA, c pGX0001 empty vector backbone, and $\mathbf{d}$ naïve muscle. Muscle tissue was harvested 3 days post DMAb injection and probed with a goat anti-humanlgG Fc antibody, followed by detection with anti-goat lgG AF88 and DAPI

a

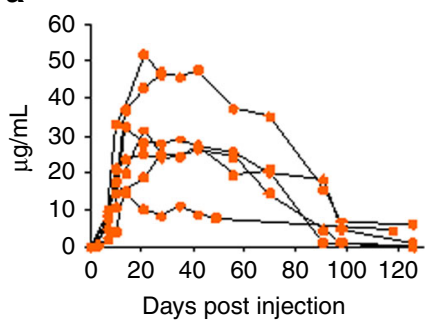

d

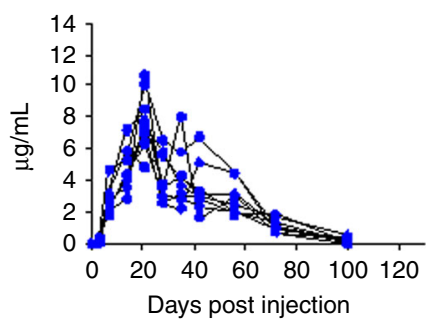

b

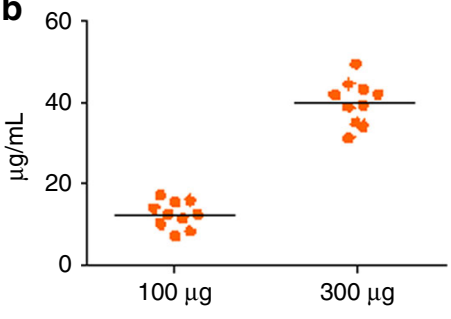

e

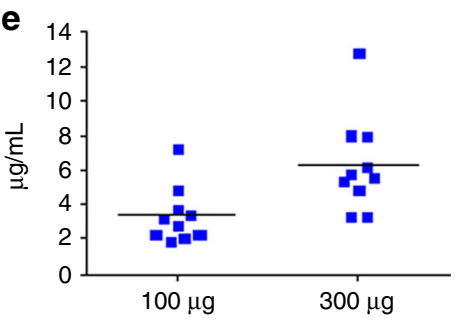

C

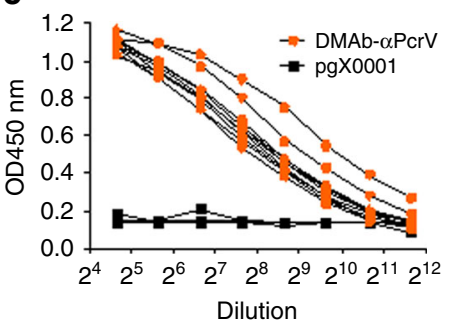

f

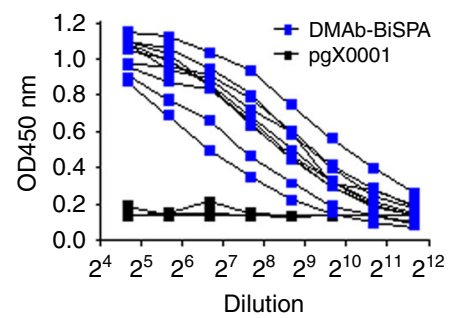

Fig. 3 In vivo expression of DMAb- $\alpha$ PcrV and DMAb-BiSPA in mice. a B6.Cg-Foxn1 < nu > /J mice ( $n=5 /$ group) were administered $100 \mu g$ of DMAb$\alpha$ PcrV by IM-EP. Serum levels of human IgG were monitored over 120 days. b Day 7 serum levels in BALB/c mice ( $n=10 /$ group) administered 100 and $300 \mu \mathrm{g}$ for DMAb- $\alpha \mathrm{PcrV}$. The line represents the mean value. c Day 7 serum binding to PcrV protein in BALB/c mice ( $n=10 / \mathrm{group})$ administered $100 \mu \mathrm{g}$ of DMAb- $\alpha$ PcrV. d B6.Cg-Foxn1 < nu > /J mice ( $n=5$ /group) were administered $100 \mu$ g of DMAb-BiSPA by IM-EP. Serum levels of human IgG were monitored over 120 days. e, b Day 7 serum levels in BALB/c mice $(n=10 /$ group) administered 100 and $300 \mu$ for DMAb-BiSPA. The line represents the mean value. $\mathbf{f}$ Day 7 serum binding to PcrV protein in BALB/c mice $(n=10 /$ group $)$ administered $100 \mu$ g of DMAb-BiSPA

immunocompromised B6.Cg-Foxn $1^{\mathrm{nu}} / \mathrm{J}$ athymic mice (nude) that lack $\mathrm{T}$ cells and have non-functional B cells. Anti-P. aeruginosa DMAb- $\alpha \mathrm{PcrV}(100 \mu \mathrm{g})$ or DMAb-BiSPA $(100 \mu \mathrm{g})$ was administered to nude mice ( $n=5 /$ group) IM in the TA or quadriceps (quad) muscles, followed by IM-EP. Serum was collected to monitor long-term human IgG1 expression in circulation. Expression of both DMAbs was observed for 100-120 days post administration, supporting the hypothesis that these novel
DNA-delivered mAbs can be produced in skeletal muscle in substantial amounts detectable in systemic circulation, with expression for several weeks (Fig. 3).

We next evaluated DMAb expression in immunocompetent $\mathrm{BALB} / \mathrm{c}$ mice as they are commonly used as a model for $P$. aeruginosa infection. Mice ( $n=10$ /group) were administered 100 and $300 \mu \mathrm{g}$ doses (three injection sites $\times 100 \mu \mathrm{g}$ ) of DMAb- $\alpha \mathrm{PcrV}$ or DMAb-BiSPA by IM-EP. The following peak DMAb 

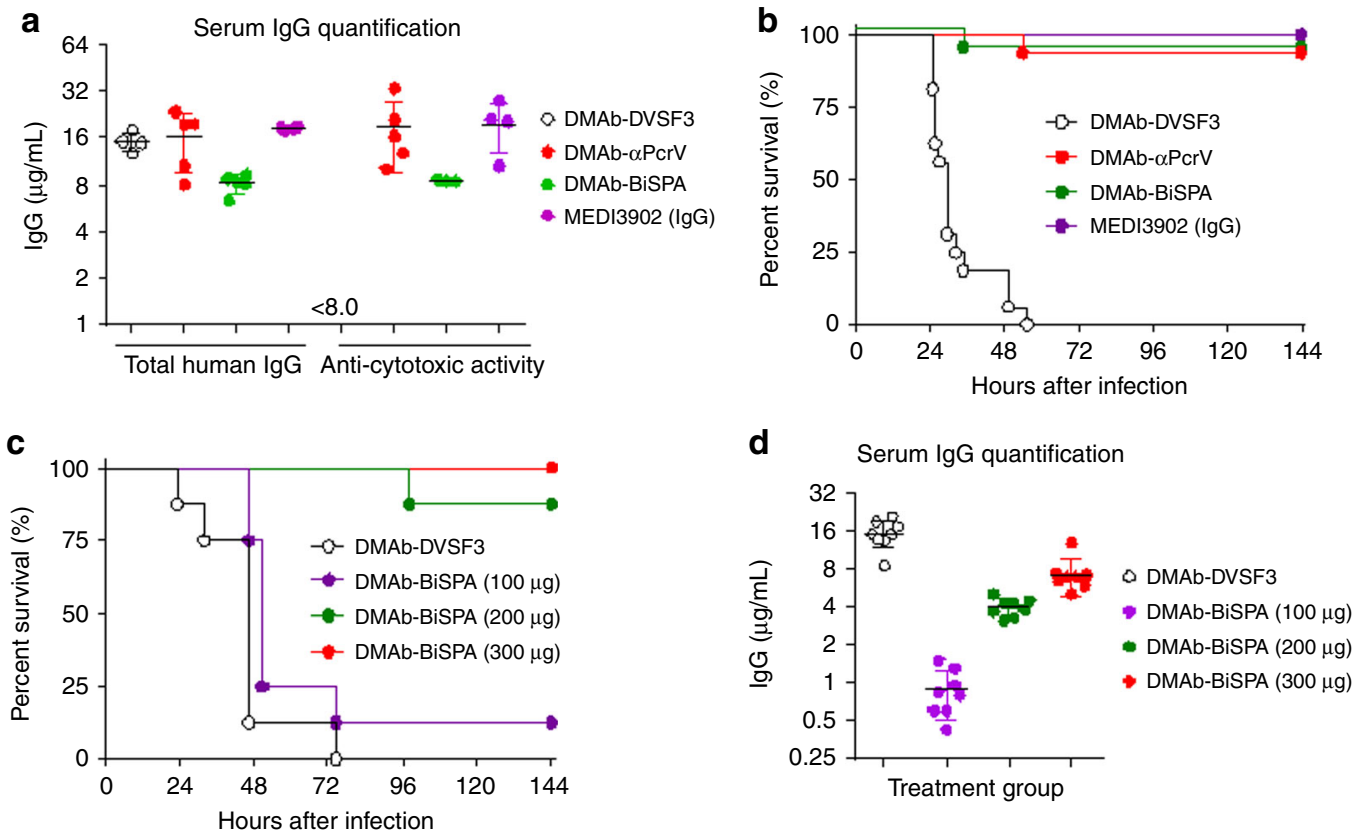

Fig. 4 Functionality and protection by DMAb- $\alpha$ PcrV and DMAb-BiSPA in mice. a BALB/c mice were administered $300 \mu$ of $D M A b-\alpha P c r V$, DMAb-BiSPA, or MEDI3902 lgG $\left(2 \mathrm{mg} \mathrm{kg}^{-1}\right) . n=5$ mice/group. Two animals from the DMAb-BiSPA were below the limit of detection of the anti-cytotoxic activity assay. Antibody levels are representative of DMAb in serum on the day of challenge. $\mathbf{b}$ In vivo protection in BALB/c mice following administration of control DMAb-DVSF3 (black open circles), DMAb- $\alpha$ PcrV (red circle), DMAb-BiSPA (green circle) or on day 5 or purified MEDI3902 mAb (purple circle) on day 1 before lethal challenge (data represented is from two independent experiments, $n=8 /$ group/experiment, total $n=16$ ). c Protection with different doses of DMAb-BiSPA: $100 \mu \mathrm{g}$ (purple circle), $200 \mu \mathrm{g}$ (green circle), $300 \mu \mathrm{g}$ (red circle), or DMAb-DVSF3 (control). $n=8$ mice/group. d Serum DMAb concentrations with different doses of DMAb-BiSPA. $n=8$ mice/group

expression levels were observed at day 7 following injection for DMAb- $\alpha$ PcrV and DMAb-BiSPA, respectively: $7.1-17.1$ and 2.9-7.2 $\mu \mathrm{g} \mathrm{ml}^{-1}$ (at the $100 \mu \mathrm{g}$ dose), and 31.2-49.7 and $3.2-12.7 \mu \mathrm{g} \mathrm{ml}^{-1}$ (at the $300 \mu \mathrm{g}$ dose) (Fig. 3b, e). Human IgG1 $\mathrm{DMAb}$ expression in BALB/c mice was eliminated by the mouse immune system by day 14 (Supplementary Fig. 1a, b). For comparison, a mouse IgG2a DMAb was also designed. This demonstrated long-term expression of $>100$ days in immunecompetent $\mathrm{BALB} / \mathrm{c}$ mice, without elimination by the immune system (Supplementary Fig. 1c). To confirm the target antigen specificity of DMAbs, the day 7 post administration serum was also assayed and confirmed for binding to recombinant PcrV protein by ELISA (Fig. 3c, f).

Evaluation of DMAb- $\alpha$ PcrV and DMAb-BiSPA in a pneumonia model. The in vitro and in vivo expression studies indicated that DMAbs form full, human IgG1 antibodies that bind to recombinant PcrV protein. To address the functional activity of in vivo DNA-delivered DMAbs, we evaluated protection against the highly pathogenic and cytotoxic $P$. aeruginosa strain 6077 (PA 6077), using a lethal mouse pneumonia infection model. Mice were injected 5 days before PA 6077 challenge with DMAb$\alpha \operatorname{PcrV}(300 \mu \mathrm{g})$, DMAb-BiSPA $(300 \mu \mathrm{g})$, or an unrelated control DMAb-DVSF3 $(300 \mu \mathrm{g})$ that targets dengue virus $(\mathrm{DENV})^{20}$. We also included a positive control group in which mice received protein MEDI3902 $\operatorname{IgG}\left(2 \mathrm{mg} \mathrm{kg}^{-1}\right) 1$ day before challenge. Randomly selected animals from DMAb- $\alpha$ PcrV-treated and DMAb-BiSPA-treated animals were euthanized to monitor DMAb expression levels in serum at the time of challenge, as well as to evaluate the potency of the expressed DMAbs. As indicated in Fig. 4a, both monospecific DMAb- $\alpha$ PcrV and bispecific DMAb-BiSPA exhibited median titers of approximately 16 and $8 \mu \mathrm{g} \mathrm{ml}^{-1}$, respectively, when quantifying total human IgG from serum. We further evaluated the potency of in vivo expressed DMAb- $\alpha$ PcrV and DMAb-BiSPA by quantifying antibody expression based on the anti-cytotoxic activity from serum. No difference was observed in the quantification methods, indicating that in vivo expressed monospecific and bispecific DMAb-IgGs are fully functional and equivalent in activity in comparison to bioprocessed IgG (Fig. 4a). The remaining animals in each group were then challenged with a lethal dose of $P$. aeruginosa by intranasal inoculation followed by monitoring of survival for 6 days post infection $(144 \mathrm{~h})$. Animals receiving the control DMAb-DVSF3 succumbed to infection within $24-55 \mathrm{~h}$. In contrast, approximately $94 \%$ of animals $(15 / 16)$ that received either DMAb- $\alpha$ PcrV or DMAb-BiSPA survived challenge (Fig. 4b, Log-rank test $p<0.0001$ in comparison with DMAb-DVSF3). As expected, all the positive control animals receiving MEDI3902 IgG $\left(2 \mathrm{mg} \mathrm{kg}^{-1}\right)$ survived challenge. In addition, treatment of mice with DMAb-BiSPA at $100 \mu \mathrm{g}(1$ site $\times 100 \mu \mathrm{g}), 200 \mu \mathrm{g}$ $(2$ sites $\times 100 \mu \mathrm{g})$, or $300 \mu \mathrm{g}(3$ sites $\times 100 \mu \mathrm{g})$ followed by infection with $P$. aeruginosa, yielded concentration-dependent survival (Fig. 4c). These results were consistent with the quantification of expressed DMAb-BiSPA in serum from these animals, in which the serum protein concentration of DMAb-BiSPA decreased with decreasing amounts of electroporated DNA (Fig. 4d).

We next sought to analyze the ability of anti-Pseudomonas DMAbs to reduce the bacterial burden in the lungs and to prevent systemic bacterial dissemination. Lungs, spleen, and kidneys were assayed $24 \mathrm{~h}$ post challenge with $P$. aeruginosa followed by quantification of colony forming units (CFUs) in each tissue. A significant reduction (Kruskal-Wallis with Dunn's multiple comparison test) in CFU lung burden was observed with DMAb-BiSPA but not DMAb- $\alpha$ PcrV-treated animals (Fig. 5a). Importantly, bacterial burden in the lungs of DMAb-BiSPAtreated animals were similar to the lung burden observed from mice treated with protein MEDI3902 IgG and both anti- 


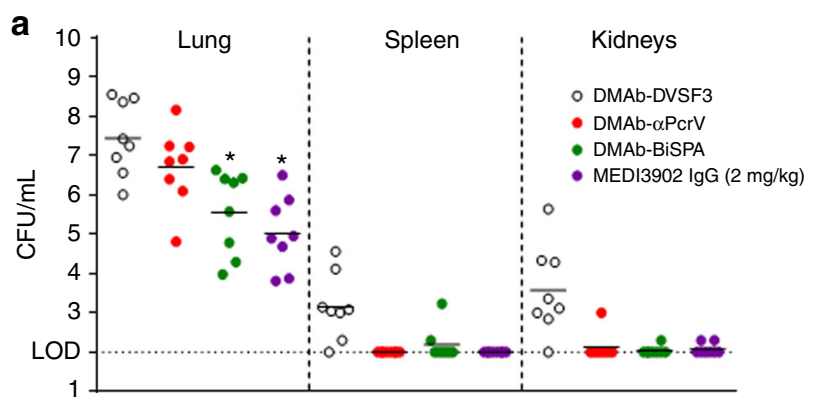

C

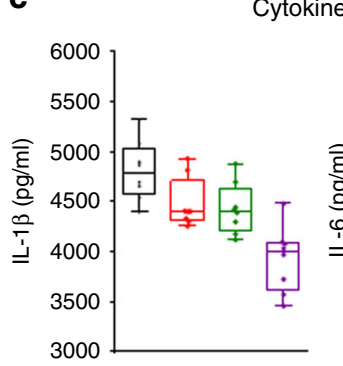

ytokine and chemokine levels in lung homogenates

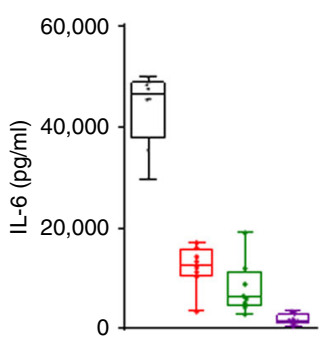

b

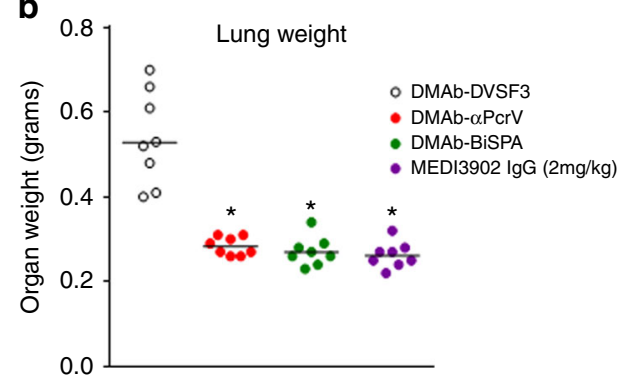

d
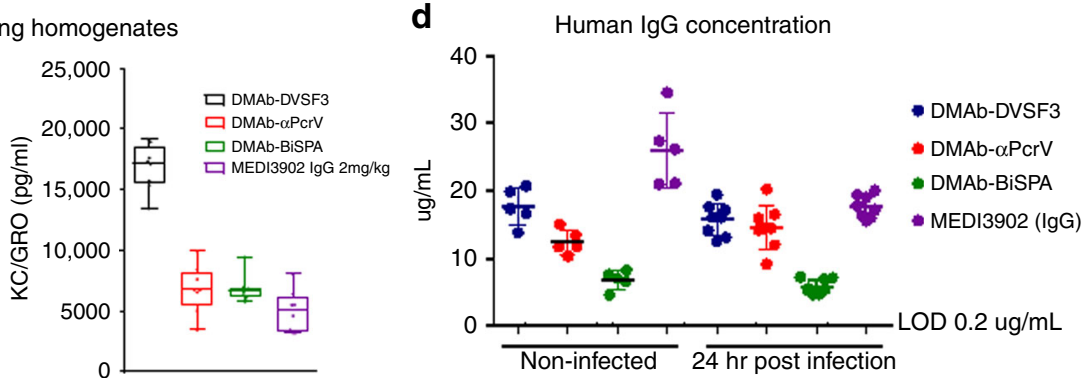

Fig. 5 Organ protective effect of DMAb- $\alpha$ PcrV and DMAb-BiSPA. a Organ burden of $P$. aeruginosa bacteria (CFU per ml) was quantified from lung, spleen, and kidneys following lethal pneumonia challenge in animals treated with DMAb-DVSF3, DMAb- $\alpha$ PcrV, DMAb-MEDI3902, or MEDI3902 IgG. b Lung weight in infected animals following DMAb-treatment. c Levels of pro-inflammatory cytokines and chemokines in lung homogenates of DMAb-treated animals following lethal challenge. a-c $n=8$ mice/group. The line represents the mean value. Box and whisker plots display all points and bars indicate minimum to maximum values. d Serum lgG levels of DMAb and MEDI3902 lgG in uninfected animals compared with infected animals at $24 \mathrm{~h}$ following lethal pneumonia challenge

Pseudomonas DMAbs reduced dissemination of bacteria to the spleen and kidneys when compared to the control DMAb-DVSF3 (Fig. 5a). In addition, DMAb- $\alpha$ PcrV, DMAb-BiSPA, and MEDI3902 IgG were effective in preventing pulmonary edema in infected animals, as measured by lung weight, compared to control DMAb-DVSF3-treated mice (Fig. 5b). Consistent with these results, proinflammatory cytokines IL-1 $\beta$ and IL- 6 as well as the chemokine KC/GRO (Fig. 5c) were also reduced in antiPseudomonas DMAb-treated and protein IgG-treated mice vs. the control DMAb-DVSF3. Serum IgG levels were compared between uninfected animals and infected animals $24 \mathrm{~h}$ post PA 6077 challenge (Fig. $5 \mathrm{~d}$ ). Taken together, these data suggest that DNAdelivered $\mathrm{mAbs}$ produced in vivo in skeletal muscle mediate protective activity and exhibit similar potency to exogenously produced IgG mAbs.

Lung histopathology following challenge. Histopathology of lungs harvested at $48 \mathrm{~h}$ post infection demonstrated a marked alveolitis in DMAb-DVSF3-treated animals with infiltrates of neutrophils and macrophages within alveolar and perivascular spaces, along with areas of hemorrhage and alveolar necrosis. In contrast, and consistent with the reduction in proinflammatory cytokines and chemokines from lung supernatants described above, there was a clear reduction in inflammation with mild populations of primarily neutrophils and fewer macrophages in DMAb-BiSPA-treated animals with similar changes seen as well as in the DMAb- $\alpha \mathrm{PcrV}$ and control MEDI3902 IgG groups (Fig. 6).

DMAb combination with antibiotics. Broad-spectrum carbapenem family antibiotics such as meropenem (MEM) are administered when a Gram negative or $P$. aeruginosa infection is suspected. Further last-resort antibiotic regimens, such as colistin, are associated with high toxicity in humans ${ }^{21,22}$ and there is the potential for the bacterium to acquire further anti-microbial resistance ${ }^{23-25}$. Therefore, alternative and adjunctive strategies to reduce these risks would be highly advantageous. We therefore evaluated the potential application of DMAb-BiSPA treatment in combination with MEM. For these experiments, we utilized a subtherapeutic dose of MEM $\left(2.3 \mathrm{mg} \mathrm{kg}^{-1}\right)$ to simulate the inadequate drug exposure encountered in patients infected with a resistant bacterium, and a subtherapeutic dose of DMAb-BiSPA $(100 \mu \mathrm{g}$, identified in Fig. 4c). Combining these subtherapeutic dosages resulted in $67 \%$ survival compared with $10 \%$ in animals that received DMAb-BiSPA alone (Log rank test $p=0.026$, Fig. 7 ). Control mice that received MEM alone or the DMAb-DVSF3 did not survive lethal challenge. Taken together, our results support the potential application of DMAb treatment as either a standalone treatment or in combination with existing antibiotic regimens. Furthermore, these data support the hypothesis that DMAb administration functions similarly to purified IgG mAbs and can mediate enhanced protective activity when combined with standard of care antibiotic treatment regimens.

\section{Discussion}

The global health and economic impact of antimicrobial resistant infections is staggering and there is a compelling need to investigate novel strategies to prevent or treat these infections. Over 2 million cases of community-acquired and hospital-acquired resistant infections are reported each year in the United States ${ }^{2}$. A large percentage of these infections are caused by ESKAPE pathogens and broad-spectrum antibiotics are frequently administered as last-resort treatment ${ }^{26}$. However, antibiotic usage is often associated with negative side effects, including disruption of the beneficial microflora and potential for the spread of crossresistance in non-targeted bacteria. Furthermore, inappropriate usage of antibiotics in humans or for agricultural purposes is likely a contributing factor to global resistance rates ${ }^{27}$. This growing predicament and the lack of novel mechanism antibiotics in the pharmaceutical pipeline has fostered the development of 


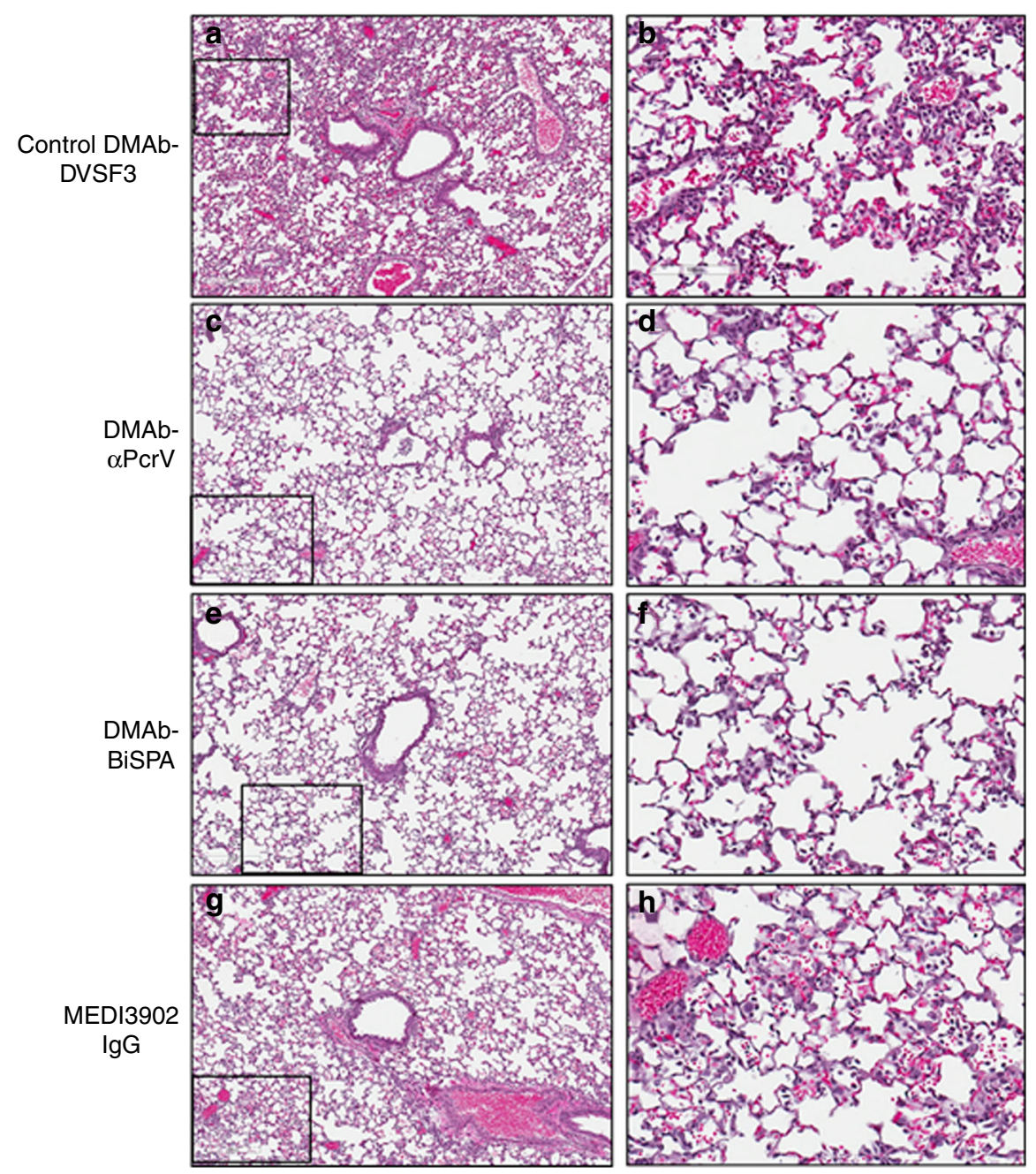

Fig. 6 Histology of acute pneumonia at $48 \mathrm{~h}$ post infection with P. aeruginosa. a Post-EP with DMAb-DVSF3 showing coalescing areas of marked alveolar infiltrate and hemorrhage $(10 \times$ magnification). b Alveoli have marked neutrophilic infiltrates, hemorrhage, and areas of necrosis (inset). c Mild pneumonia and occasional bronchiolar debris with DMAb- $\alpha \mathrm{PcrV}(10 \times$ magnification $)$. d Alveolar infiltrates comprises mixed neutrophilic and macrophage populations (inset). e DMAb-BiSPA group with mild alveolitis $(10 \times$ magnification). $\mathbf{f}$ Primarily neutrophilic infiltrates and mild hemorrhage in alveolar spaces (inset). $\mathbf{g}$ MEDI3902 IgG control demonstrates moderate alveolitis $(10 \times$ magnification). h Alveolar spaces contain neutrophils admixed with cellular debris and hemorrhage (inset). Representative data from five mice/group

alternative antimicrobial strategies such as pathogen-specific mAbs. We previously reported on the derivation of a monospecific anti-PcrV mAb (V2L2MD) and bispecific anti-PcrV/Psl mAb MEDI3902 that confer potent protective activity against one of the ESKAPE pathogens, $P$. aeruginos $a^{16,17}$. Using these clones as model molecules, we encoded the IgG heavy and light chain sequences into a synthetic DNA vector to test the feasibility of plasmid DNA delivery (DMAb) as an alternative mode of direct delivery of $\mathrm{mAb}$ in vivo to overcome some of the limitations of traditional $\mathrm{mAb}$ manufacturing and delivery impeding broader use.

In the current study, we demonstrated that mAbs against $P$. aeruginosa can be encoded in synthetic DNA vectors, DMAbs, and produced in vivo by skeletal muscle. The anti-Pseudomonas DMAbs bound effectively to therapeutic targets and were protective in a mouse model of lethal pneumonia caused by an aggressive $P$. aeruginosa strain. We identified that a single dose of $\mathrm{DMAb}$ is transiently expressed for 3-4 months and protection against lethal infection is comparable to treatment of mice with purified IgG. This result supports the possibility of long-term $\mathrm{mAb}$ administration, as DMAbs can be continuously expressed from muscle until the plasmid is eventually lost. In addition to routine administration, another foreseeable advantage for anti- $P$. aeruginosa DMAbs would be for high-risk patients with recurring infections related to chronic illnesses or implanted devices, where DMAbs may reduce the need for extended antibiotic regimens. Furthermore, we demonstrated that DMAbs can also function synergistically with a commonly used antibiotic, MEM. The synergistic effect of DMAb and antibiotic combination suggests that this strategy could have potential in reducing antibiotic treatment regimens, thereby reducing the length of antibiotic exposure in patients. This adjunctive activity is equivalent to that observed with protein $\operatorname{IgG}$ in previous studies $^{18}$. Although we did not evaluate DMAb activity against a panel of $P$. aeruginosa bacterial strains, the experiments outlined in this manuscript demonstrate that DMAb is functionally equivalent to protein IgG. All bioprocessed anti-Pseudomonal IgG mAbs (anti-Psl, anti-PcrV, and MEDI3902) have been previously shown to be protective against $P$. aeruginosa clinical isolates derived from diverse serotypes, multiple T3S phenotypes (cytotoxic vs. invasive strain; $\mathrm{ExoU}^{+}, \mathrm{ExoS}^{-}$; ExoU ${ }^{-}, \mathrm{ExoS}^{+}$, respectively), and multiple infection sites ${ }^{16-18,28,29}$.

The field of $\mathrm{mAb}$ engineering is evolving dynamically and DMAb delivery offers an additional strategy to help transport 
a

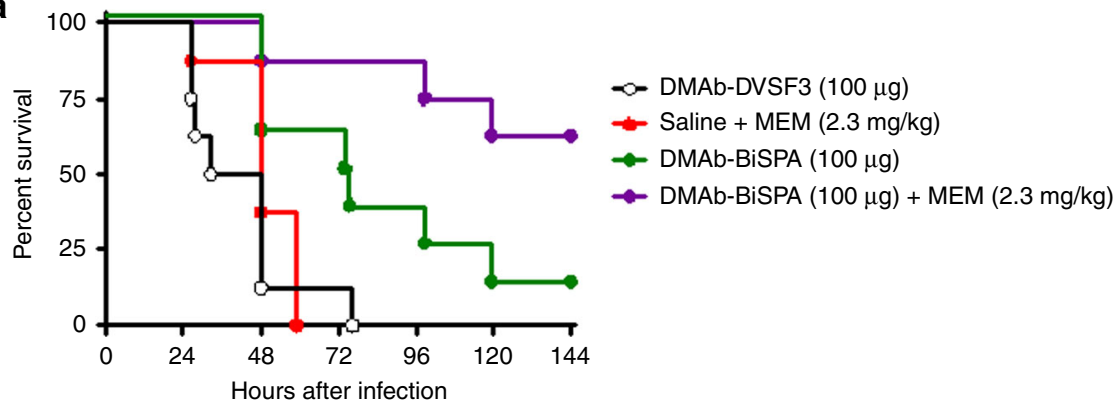

b

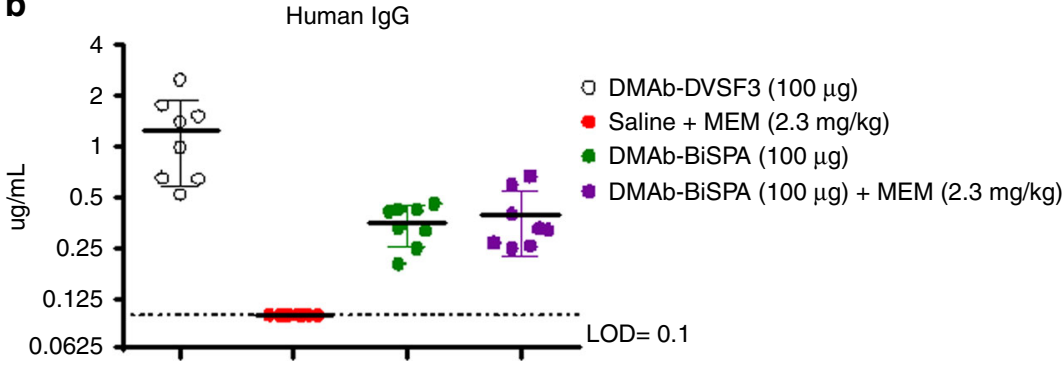

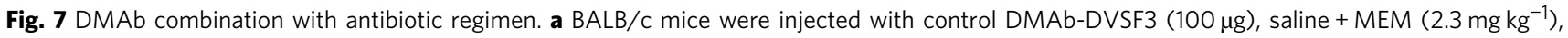
DMAb-BiSPA $(100 \mu \mathrm{g})$, or DMAb-BiSPA $(100 \mu \mathrm{g})+\operatorname{MEM}\left(2.3 \mathrm{mg} \mathrm{kg}^{-1}\right)$ and then challenged with a lethal dose of $P$. aeruginosa 6077 . MEM was administered $1 \mathrm{~h}$ post lethal challenge. Animals were monitored for $144 \mathrm{~h}$ post infection. $n=8$ mice/group. $\mathbf{b}$ DMAb serum levels in animals before lethal challenge. $n=8$ mice/group. The line represents the mean value and error bars represent standard deviation

biologically functional $\mathrm{mAbs}$ rapidly in vivo. In addition to obvious clinical benefits, in vivo expression of non-traditional bispecific mAb isoforms, as presented here, emphasizes the versatility of muscle to be engaged as protein production factories. Importantly, DMAb expression is transient, with similar efficacy to other therapeutic deliveries. It may be possible to develop an inducible system that will eliminate the DNA plasmid when it is no longer needed. Alternatively, DMAb DNA can potentially be re-administered indefinitely as there are no associated anti-vector responses, allowing for long-term therapy through repeat administration $^{30-32}$. We believe that DMAb delivery represents a significant advancement not only for mAb therapy and DNA-delivery technology, but also for novel pathogen-specific treatment approaches to enhance host immunity.

Recently delivery of DNA-encoded antibodies that target Her2 in a mouse model of human breast cancer carcinoma ${ }^{33}$ has been reported. This study demonstrated anti-tumor efficacy comparable to protein IgG, further supporting the concept that a geneencoded $\mathrm{mAb}$ can have functionality. To our knowledge, our study is the first demonstration of DNA-encoded mAb delivery that is protective against a bacterial target and the first delivery of an engineered IgG isoform. Early studies with DNA plasmidencoded antibodies demonstrated feasibility but exhibited lowIgG expression in serum ${ }^{34}, 35$. Our group previously demonstrated the protective efficacy of DMAbs targeting viral infections, showing rapid protection against chikungunya ${ }^{36}$ and DENV infections ${ }^{20}$. The DMAb-targeting DENV did not promote antibody-dependent enhancement of disease. These two infectious disease models did not require high serum IgG levels; however, optimized DMAb formulations to increase expression levels are desirable so as to provide extended coverage after a single DMAb administration. Toward this end, we sought to optimize serum DMAb expression levels for our efforts against $P$. aeruginosa (Supplementary Fig. 2). This work included the inclusion of hyaluronidase into the formulation regimen, which allowed for greater IgG expression from the treated muscle.
Although further study is required for translation to humans, DMAbs are a step toward enabling routine delivery of $\mathrm{mAb}$, with the potential for increasing accessibility to diverse communities worldwide. Dose translation in larger animals and humans will be important to address in future studies, particularly understanding DNA dose limitations during DMAb administration. This includes investigating different delivery and formulation optimizations that will enhance DNA expression in vivo. One strategy may be to employ other extracellular matrix enzymes to facilitate DNA entry into muscle cells ${ }^{37}$. Further study in non-human primates may help to understand the threshold for DNA dosage and impact on pharmacokinetic levels. Additional studies evaluating the glycosylation patterns of human IgG DMAbs produced in muscle would be beneficial to compare with bioprocessed protein IgG; however, in the context of the current study there was no difference in functionality between DMAb and its protein IgG counterpart.

In conclusion, our work described herein could be of significance for the treatment of AMR infections, particularly against ESKAPE pathogens that are refractory to many broad-spectrum antibiotic regimens. The DMAb strategy is versatile and can deliver monospecific IgGs against multiple antigenic targets as well as novel bispecific IgGs. The sustained serum mAb trough levels produced by a single dose of DMAb are consistent with functionality and protective levels afforded by bioprocessed protein IgG in vivo. The rapid development of this platform and prolonged transient expression from muscle are favorable in comparison with protein IgG $\mathrm{mAb}$ regimens as it could enable less frequent $\mathrm{mAb}$ administration. Furthermore, DMAb-encoding DNA is temperature stable allowing for transport, long-term storage, and administration to broader populations. These attractive features combined with the safety profile of DNA delivery in humans, support further DMAb studies in larger animal models as a pathogen-specific approach to targeting infectious diseases and other potential therapeutic targets. 


\section{Methods}

Cell lines and bacteria. Human embryonic kidney (HEK) 293 T cells (ATCC CRL-3216) were maintained in Dulbecco's Modified Eagle's Medium (Gibco, Life Technologies, Carlsbad, CA, USA), supplemented with $10 \%$ fetal bovine serum (FBS, Atlas Biologicals, Fort Collins, CO, USA), in mycoplasma-free conditions. Routine testing was performed at the University of Pennsylvania. All cells were maintained at a low passage number. P. aeruginosa keratitis clinical isolate 6077 (PA 6077-provided by Dr. Joanna Goldberg-Emory University) ${ }^{38}$ is a cytotoxic $\left(\mathrm{ExoU}^{+}\right)$strain that was used for all infection experiments.

DMAb construction and expression. The sequences of the single specificity antiPcrV IgG (clone V2L2MD) and engineered bispecific IgG (dual specificity for PcrV and Psl, clone MEDI3902) were obtained as previously described ${ }^{17}, 18$. The nucleotide sequence for each human IgG1 heavy and Igk light chains were codon optimized for both mouse and human biases to enhance expression in mammalian cells $^{39,40}$. Sequences were also RNA optimized for improved mRNA stability. This has been shown to result in more efficient translation on the ribosome ${ }^{41,42}$, leading to increase protein yield ${ }^{43}$. The optimized heavy and light chain genes were then inserted into the pGX0001 DNA expression vector, under the control of a human cytomegalovirus promoter and bovine growth hormone polyA

Both genes were encoded in cis, separated by a furin cleavage site (RGRKRRS) and P2A peptide. The result was two plasmids: DMAb- $\alpha \mathrm{PcrV}$ and DMAb-BiSPA. HEK 293T cells were transfected with DMAb DNA using GeneJammer (Agilent, Wilmington, DE) transfection reagent. Cell supernatants and cell lysates were harvested $48 \mathrm{~h}$ post transfection and assayed for human IgG production by ELISA and Western blot.

Mouse muscle tissue immunofluorescence. $\mathrm{BALB} / \mathrm{c}$ mice were injected with $100 \mu \mathrm{g}$ of DMAb by IM injection in the TA muscle followed by IM-EP. Tissue was harvested 3 days post injection, fixed in $4 \%$ Neutral-buffered Formalin (BBC Biochemical, Washington State) and immersed in 30\% (w/v) sucrose (Sigma, MO) in D.I. water. Tissues were then embedded into O.C.T. compound (Sakura Finetek, $\mathrm{CA}$ ) and snap-frozen. Frozen tissue blocks were sectioned to a thickness of $18 \mu \mathrm{m}$. Muscle sectioned were incubated with Blocking-Buffer $(0.3 \%(\mathrm{v} / \mathrm{v})$ Triton-X (Sigma), 2\% (v/v) donkey serum in PBS) for $30 \mathrm{~min}$, covered with Parafilm. Goat anti-human IgG-Fc fragment antibody (A-80-104A, Bethyl, TX) was diluted 1:100 in incubation buffer $(1 \%(\mathrm{w} / \mathrm{v})$ BSA (Sigma), $2 \%(\mathrm{v} / \mathrm{v})$ donkey serum, $0.3 \%(\mathrm{v} / \mathrm{v})$ Triton-X (Sigma), and $0.025 \%(\mathrm{v} / \mathrm{v}) 1 \mathrm{~g} \mathrm{ml}^{-1}$ Sodium Azide (Sigma) in PBS). $50 \mu \mathrm{l}$ of staining solution was added to each section and incubated for $2 \mathrm{~h}$. Sections were washed $5 \mathrm{~min}$ in $1 \times$ PBS three times. Donkey anti-goat IgG AF488 (ab150129, Abcam, USA) was diluted 1:200 in incubation buffer and 50 $\mathrm{\mu l}$ was added to each section. Section were washed after $1 \mathrm{~h}$ incubation and mounted with DAPIFluoromount (SouthernBiotech, AL) and covered.

In vivo expression of DMAb constructs was imaged with a BX51 Fluorescent microscope (Olympus) equipped with Retiga3000 monochromatic camera (QImaging).

Human IgG quantification by ELISA and by anti-cytotoxic activity. Ninety-sixwell, high-binding immunosorbent plates were coated with $10 \mu \mathrm{gl}^{-1}$ purified anti-human IgG-Fc (A-80-104A, Bethyl Laboratories, Montgomery, TX) and incubated overnight at $4{ }^{\circ} \mathrm{C}$. The following day, plates were washed and blocked at room temperature for at least $1 \mathrm{~h}$ with PBS containing $10 \%$ FBS. Samples were serially diluted two-fold and transferred to the blocked plate and incubated for $1 \mathrm{~h}$ at room temperature. Purified human IgGk (P80-111, Bethyl Laboratories, Montgomery, TX) was used as a standard. Following incubation, samples were probed with an anti-human IgGK antibody conjugated to horseradish peroxidase (A80115P, Bethyl Laboratories, Montgomery, TX) at a 1:20,000 dilution. Plates were developed using o-Phenylenediamine dihydrochloride substrate (OPD, Sigma Aldrich, St. Louis, $\mathrm{MO}$ ) and stopped with $2 \mathrm{~N} \mathrm{H}_{2} \mathrm{SO}_{4}$. A BioTek Synergy2 plate reader (Bioteck, Winooski, VT) was used to read the plates at OD450 nm. Alternatively, human IgG from serum was quantified as described above with the exception of using an anti-idiotype mAb $(0.05 \mu \mathrm{g}$ per well suspended in $0.2 \mathrm{M}$ sodium bicarbonate buffer, $\mathrm{pH}$ 9.4) specific for V2L2MD or MEDI3902 as the capture reagent. Purified V2L2MD or MEDI3902 was used as a standard.

DMAb was also quantified from serum using 384-well black MaxiSorp plates (Nalge, Nunc, Thermo Fisher Scientific, Waltham, MA) coated with $10 \mu \mathrm{g} \mathrm{ml}^{-1}$ Goat anti-Human IgG $(\mathrm{H}+\mathrm{L})$ (31410, Pierce, Thermo Fisher). Plates were washed and blocked for 1-2 $\mathrm{h}$ at room temperature with Blocker Casein in PBS (Thermo Fisher). After blocking, a standard containing MEDI3902 or V2L2MD was serially diluted $1: 2$ across the plate, while serum samples were diluted 1:20, 1:40, 1:80, and $1: 160$. Plates containing the samples were then incubated for $1 \mathrm{~h}$ at room temperature. After washing, plates were probed with Donkey anti-Human IgG-HRP (709-035-149, JacksonImmuno Research, West Grove, PA) at a 1:4000 dilution and incubated for $1 \mathrm{~h}$ at room temperature. After washing, the immune reaction was developed by adding SuperSignal ELISA Pico Reagent (Thermo Fisher) and fluorescence was read on the Perkin Elmer Envision.

$\mathrm{DMAb}$ was also quantified from serum based on anti-cytotoxic activity mediated by DMAb- $\alpha$ PcrV and DMAb-BiSPA that measures the protection of A549 cells (ATCC ${ }^{\circledR}$ CCL-185 $185^{\circ)}$ from the cytotoxic effects of PA 6077 . The activity of mouse serum was compared to a standard curve of naïve mouse serum spiked with V2L2MD IgG.
Binding ELISA. Ninety-six-well plates immunosorbent plates were coated overnight with Pseudomonas PcrV protein (MedImmune, Gaithersburg, MD) at $0.5 \mu \mathrm{g}$ $\mathrm{ml}^{-1}$. The following day, serum samples from DMAb-administered animals were serially diluted two-fold and then transferred to the blocked plate. Samples were probed with an anti-human IgG H + L antibody conjugated to HRP (SAB3701359, Sigma Aldrich, St. Louis, MO) at a dilution of 1:5000 and developed with OPD substrate.

Western blot. The cell lysates from DMAb-transfected cells were collected in cell lysis buffer (Cell Signalling Technologies, Danvers, MA). Samples were centrifuged at $16,000 \times g$ and the supernatant containing the protein fraction was collected. The samples were quantified using a bicinchoninic acid assay (Pierce, Thermo Fisher Scientific) and $10 \mu \mathrm{g}$ total lysate was loaded on a $4-12 \%$ Bis-Tris SDS-PAGE gel (NuPAGE, Thermo Fisher Scientific). The gel was transferred to a nitrocellulose membrane using the iBlot2 system (Thermo Fisher Scientific). The membrane was blocked in $5 \%$ powdered skim milk $+0.5 \%$ Tween- 20 and then probed using a donkey anti-human $\mathrm{H}+\mathrm{L}$ antibody conjugated to HRP (SAB3701359, Sigma Aldrich, 1:5000 dilution). Bands were developed using a chemiluminescent system (ECL, GE Healthcare, UK) and visualized on film.

Mice. Female, 6-8-week-old B6.Cg-Foxn1nuJ and BALB/c mice were purchased from The Jackson Laboratory (Bar Harbor, ME) and housed in the animal facilities at the University of Pennsylvania or MedImmune, AstraZeneca. All animal protocols were approved by the institutional University of Pennsylvania and MedImmune Institutional Animal Care and Use Committees (IACUC), following guidelines from Association for Assessment and Accreditation of Laboratory Animal Care International. Further IACUC oversight was provided by The Animal Care and Use Review Office, required for DARPA-funded research. Animals received an IM pre-injection of hyaluronidase ( $400 \mathrm{U} \mathrm{ml}^{-1}$, Sigma Aldrich) 30 min- $1 \mathrm{~h}$ before IM injection of $100-300 \mu \mathrm{g}$ DMAb- $\alpha \mathrm{PcrV}$ or DMAb-BiSPA in the TA or quad muscles, followed by electroporation (IM-EP). Serum levels of DMAbs were monitored following administration.

Lethal pneumonia challenge. BALB/c mice ( $n=8 /$ group) received $100 \mu \mathrm{g}$ or $300 \mu \mathrm{g}$ of DMAb- $\alpha$ PcrV or DMAb-BiSPA by IM-EP at day 5 before challenge. The unrelated DENV DMAb-DVSF $3^{20}$ was included as a control. A fourth group of animals received an intraperitoneal injection of purified protein IgG MEDI3902 $\left(2 \mathrm{~m} \mathrm{~kg}^{-1}\right)$ on day -1 before challenge. On day 0 , animals received an intranasal challenge of 9.75e5-1.0e6 CFUs of the aggressive, anti-microbial resistant P. aeruginosa strain 6077 . Animals were monitored for 6 days following intranasal challenge for survival as described in ref. ${ }^{16}$. Briefly, animals were anesthetized with ketamine and xylazine followed by intranasal administration of the bacterial inoculum contained in $0.05 \mathrm{ml}$. For organ burden analyses, lungs, spleens, and livers were harvested from DMAb-treated animals $24 \mathrm{~h}$ post infection followed by homogenizing and plating of Luria agar plates for enumeration of bacterial CFU. IL-1 $\beta$, IL-6, and KC/GRO were quantified from the supernatant of lung homogenates using a multiplex kit (Meso Scale Diagnostics), according to the manufactures instructions. For DMAb and MEM combination experiments, MEM was administered subcutaneously $4 \mathrm{~h}$ after infection.

Histopathology. Lungs were harvested at $48 \mathrm{~h}$ post infection and fixed in $10 \%$ neutral buffered formalin (VWR, Radnor, PA) for a minimum of $48 \mathrm{~h}$. Fixed tissues were then routinely processed and embedded in paraffin, sectioned at 3- $\mu \mathrm{m}$ thickness, and stained with Gill's hematoxylin (Mercedes Medical, Sarasota, FL) and eosin (Surgipath, Richmond, IL) for histologic evaluation by a pathologist blinded to the experimental conditions.

Statistics. All statistical analyses were peformed using GraphPad Prism 6.0 software (La Jolla, CA) or SPSS (IBM). Sample size calculations for two independent proportions were calculated with alpha 0.05 and power 0.90 . A minimum of $n=5$ mice was calculated to be needed in order to ensure adequate power. We performed student's $t$-test or one-way analysis of variance (ANOVA) calculations, where necessary. Survival data was represented by a Kaplan-Meier survival curve and significance was calculated using a log-rank test and one-way ANOVA with correction for multiple comparisons. These data were considered significant if $p<$ 0.05 . The lines in all graphs represent the mean value and error bars represent the standard deviation. No samples or animals were excluded from the analysis. Randomization was not performed for the animal studies. Samples and animals were not blinded before performing each experiment.

Data availability. The nucleotide sequences for the optimized human IgG1 heavy and Igk light chains have been deposited in the NCBI Nucleotide database with accession codes LQ055727.1, LQ055728.1, LQ055748.1, and LQ055750.1. The authors declare that all other relevant data supporting the findings of the study are available in this article and its Supplementary Information files, or from the corresponding author upon request. 
Received: 4 January 2017 Accepted: 10 July 2017

Published online: 21 September 2017

\section{References}

1. O'Neill, J. Tackling drug-resistant infections globally: final report and recommendations, https://amr-review.org/sites/default/files/160518_Final\% 20paper_with\%20cover.pdf (2016).

2. CDC. Antibiotic resistance threats in the United States, http://www.cdc.gov/ drugresistance/threat-report-2013/ (2013).

3. Matera, M. G., Page, C., Rogliani, P., Calzetta, L. \& Cazzola, M. Therapeutic monoclonal antibodies for the treatment of chronic obstructive pulmonary disease. Drugs 76, 1257-1270 (2016).

4. Allison, T. L. Immunosuppressive therapy in transplantation. Nurs. Clin. North Am. 51, 107-120 (2016).

5. Chan, A. C. \& Carter, P. J. Therapeutic antibodies for autoimmunity and inflammation. Nat. Rev. Immunol. 10, 301-316 (2010).

6. Nelson, A. L., Dhimolea, E. \& Reichert, J. M. Development trends for human monoclonal antibody therapeutics. Nat. Rev. Drug Discov. 9, 767-774 (2010).

7. Scott, A. M., Wolchok, J. D. \& Old, L. J. Antibody therapy of cancer. Nat. Rev. Cancer 12, 278-287 (2012).

8. Weiner, L. M., Surana, R. \& Wang, S. Monoclonal antibodies: versatile platforms for cancer immunotherapy. Nat. Rev. Immunol. 10, 317-327 (2010).

9. Zumla, A. et al. Host-directed therapies for infectious diseases: current status, recent progress, and future prospects. Lancet Infect. Dis. 16, e47-e63 (2016).

10. Ter Meulen, J. Monoclonal antibodies in infectious diseases: clinical pipeline in 2011. Infect. Dis. Clin. North Am. 25, 789-802 (2011).

11. Sardesai, N. Y. \& Weiner, D. B. Electroporation delivery of DNA vaccines: prospects for success. Curr. Opin. Immunol. 23, 421-429 (2011).

12. Byrd, M. S. et al. Genetic and biochemical analyses of the Pseudomonas aeruginosa Psl exopolysaccharide reveal overlapping roles for polysaccharide synthesis enzymes in Psl and LPS production. Mol. Microbiol. 73, 622-638 (2009).

13. Jackson, K. D., Starkey, M., Kremer, S., Parsek, M. R. \& Wozniak, D. J. Identification of psl, a locus encoding a potential exopolysaccharide that is essential for Pseudomonas aeruginosa PAO1 biofilm formation. J. Bacteriol. 186, 4466-4475 (2004).

14. Mishra, M. et al. Pseudomonas aeruginosa Psl polysaccharide reduces neutrophil phagocytosis and the oxidative response by limiting complementmediated opsonization. Cell Microbiol. 14, 95-106 (2012).

15. Hauser, A. R. The type III secretion system of Pseudomonas aeruginosa: infection by injection. Nat. Rev. Microbiol. 7, 654-665 (2009).

16. DiGiandomenico, A. et al. Identification of broadly protective human antibodies to Pseudomonas aeruginosa exopolysaccharide Psl by phenotypic screening. J. Exp. Med. 209, 1273-1287 (2012).

17. Warrener, P. et al. A novel anti-PcrV antibody providing enhanced protection against Pseudomonas aeruginosa in multiple animal infection models. Antimicrob. Agents Chemother. 58, 4384-4391 (2014).

18. DiGiandomenico, A. et al. A multifunctional bispecific antibody protects against Pseudomonas aeruginosa. Sci. Transl. Med. 6, 262ra155 (2014).

19. Ferraro, B. et al. Clinical applications of DNA vaccines: current progress. Clin. Infect. Dis. 53, 296-302 (2011).

20. Flingai, S. et al. Protection against dengue disease by synthetic nucleic acid antibody prophylaxis/immunotherapy. Sci. Rep. 5, 12616 (2015)

21. Falagas, M. E. et al. Toxicity after prolonged (more than four weeks) administration of intravenous colistin. BMC Infect. Dis. 5, 1 (2005).

22. Lim, L. M. et al. Resurgence of colistin: a review of resistance, toxicity, pharmacodynamics, and dosing. Pharmacotherapy 30, 1279-1291 (2010).

23. Hirsch, E. B. \& Tam, V. H. Impact of multidrug-resistant Pseudomonas aeruginosa infection on patient outcomes. Expert Rev. Pharmacoecon. Outcomes Res. 10, 441-451 (2010).

24. Lister, P. D., Wolter, D. J. \& Hanson, N. D. Antibacterial-resistant Pseudomonas aeruginosa: clinical impact and complex regulation of chromosomally encoded resistance mechanisms. Clin. Microbiol. Rev. 22, 582-610 (2009)

25. Breidenstein, E. B., de la Fuente-Nunez, C. \& Hancock, R. E. Pseudomonas aeruginosa: all roads lead to resistance. Trends Microbiol. 19, 419-426 (2011).

26. Rice, L. B. Federal funding for the study of antimicrobial resistance in nosocomial pathogens: no ESKAPE. J. Infect. Dis. 197, 1079-1081 (2008).

27. Collignon, P. C. et al. World Health Organization ranking of antimicrobials according to their importance in human medicine: a critical step for developing risk management strategies to control antimicrobial resistance from food animal production. Clin. Infect. Dis. 63, 1087-1093 (2016).
28. Thaden, J. T. et al. Pseudomonas aeruginosa bacteremic patients exhibit nonprotective antibody titers against therapeutic antibody targets PcrV and Psl exopolysaccharide. J. Infect. Dis. 213, 640-648 (2016).

29. Zegans, M. E. et al. Association of biofilm formation, Psl exopolysaccharide expression, and clinical outcomes in Pseudomonas aeruginosa keratitis: analysis of isolates in the steroids for corneal ulcers trial. JAMA Ophthalmol. 134, 383-389 (2016).

30. Hirao, L. A. et al. Comparative analysis of immune responses induced by vaccination with SIV antigens by recombinant Ad5 vector or plasmid DNA in rhesus macaques. Mol. Ther. 18, 1568-1576 (2010).

31. Williams, J. A. Vector design for improved DNA vaccine efficacy, safety and production. Vaccines 1, 225-249 (2013).

32. Schmaljohn, C. S., Spik, K. W. \& Hooper, J. W. DNA vaccines for HFRS laboratory and clinical studies. Virus Res. 187, 91-96 (2014).

33. Kim, H. et al. Gene therapy using plasmid DNA-encoded anti-HER2 antibody for cancers that overexpress HER2. Cancer Gene Ther. 23, 341-347 (2016).

34. Tjelle, T. E. et al. Monoclonal antibodies produced by muscle after plasmid injection and electroporation. Mol. Ther. 9, 328-336 (2004).

35. Perez, N. et al. Regulatable systemic production of monoclonal antibodies by in vivo muscle electroporation. Genet. Vaccine Ther. 2, 2 (2004).

36. Muthumani, K. et al. Rapid and long-term immunity elicited by DNA-encoded antibody prophylaxis and DNA vaccination against chikungunya virus. J. Infect. Dis. 214, 369-378 (2016).

37. Cemazar, M. et al. Hyaluronidase and collagenase increase the transfection efficiency of gene electrotransfer in various murine tumors. Hum. Gene Ther. 23, 128-137 (2012).

38. Preston, M. J. et al. Rapid and sensitive method for evaluating Pseudomonas aeruginosa virulence factors during corneal infections in mice. Infect. Immun. 63, 3497-3501 (1995).

39. Graf, M., Deml, L. \& Wagner, R. Codon-optimized genes that enable increased heterologous expression in mammalian cells and elicit efficient immune responses in mice after vaccination of naked DNA. Methods Mol. Med. 94, 197-210 (2004).

40. Deml, L. et al. Multiple effects of codon usage optimization on expression and immunogenicity of DNA candidate vaccines encoding the human immunodeficiency virus type $1 \mathrm{Gag}$ protein. J. Virol. 75, 10991-11001 (2001).

41. Schneider, R., Campbell, M., Nasioulas, G., Felber, B. K. \& Pavlakis, G. N. Inactivation of the human immunodeficiency virus type 1 inhibitory elements allows Rev-independent expression of Gag and Gag/protease and particle formation. J. Virol. 71, 4892-4903 (1997).

42. Andre, S. et al. Increased immune response elicited by DNA vaccination with a synthetic gp120 sequence with optimized codon usage. J. Virol. 72, 1497-1503 (1998).

43. Fath, S. et al. Multiparameter RNA and codon optimization: a standardized tool to assess and enhance autologous mammalian gene expression. PLoS ONE 6, e17596 (2011).

\section{Acknowledgements}

The studies described in this manuscript were funded by a grant from the Defense Advanced Projects Research Agency (DARPA).

\section{Author contributions}

A.P. and A.D. designed and performed experiments, analyzed the data, and prepared the manuscript. A.E.K., D.H.P., K.S., J.M., L.I.C., L.W.-R. performed experiments. T.R.F.S. and S.R. performed experiments and analyzed the data. S.T.C.E., K.E.B., M.C.W., J.Y., J.J., S.F., A.S.K., K.M., L.H. aided in experimental design, data analysis, and manuscript preparation. C.K.S. and N.Y.S. experimental design, data analysis, and manuscript preparation. D.B.W. designed experiments, analyzed data, and oversaw final manuscript preparation.

\section{Additional information}

Supplementary Information accompanies this paper at doi:10.1038/s41467-017-00576-7.

Competing interests: K.M. reports receiving grants from DARPA and Inovio, receiving consulting fees from Inovio related to DNA vaccine development, and a pending patent application (to Inovio) for delivery of DNA-encoded monoclonal antibodies (PCT Patent Application No. PCT/US17/31449, "DNA Antibody Constructs for Use against Pseudomonas aeuruginosa”). T.R.F.S., S.R., J.M., K.E.B., K.S., J.Y., J.J., A.S.K., L.H., and N.Y.S. are employees of Inovio Pharmaceuticals and as such receive salary and benefits, including ownership of stock and stock option, from the company. D.B.W. has received grant funding, participates in industry collaborations, has received speaking honoraria, and has received fees for consulting, including serving on scientific review committees and board services. Remuneration received by D.B.W. includes direct payments or stock or stock options, and in the interest of disclosure he notes potential conflicts associated with this work with Inovio and possibly others. In addition, he has a patent DNA vaccine delivery pending to Inovio (see above). A.D., A.E.K., L.I.C., L.W.-R., C.K.S. are employees 
of MedImmune/AstraZeneca and may own stock in the company. All remaining authors declare no competing financial interests.

Reprints and permission information is available online at http://npg.nature.com/ reprintsandpermissions/

Publisher's note: Springer Nature remains neutral with regard to jurisdictional claims in published maps and institutional affiliations.

cc) (i) Open Access This article is licensed under a Creative Commons Attribution 4.0 International License, which permits use, sharing, adaptation, distribution and reproduction in any medium or format, as long as you give appropriate credit to the original author(s) and the source, provide a link to the Creative Commons license, and indicate if changes were made. The images or other third party material in this article are included in the article's Creative Commons license, unless indicated otherwise in a credit line to the material. If material is not included in the article's Creative Commons license and your intended use is not permitted by statutory regulation or exceeds the permitted use, you will need to obtain permission directly from the copyright holder. To view a copy of this license, visit http://creativecommons.org/ licenses/by/4.0/.

(C) The Author(s) 2017 